\title{
Basic Leucine zipper (bZIP) transcription factor genes and their response to drought stress in ginseng, Panax ginseng C.A. Meyer
}

Hongjie Li ( $\sim 2363893270 @ q q . c o m$ )

jilinnongyedaxue https://orcid.org/0000-0003-3537-673X

Jing Chen

Jilin Agricultural University

Qi Zhao

Jilin Agricultural University

Yilai Han

Jilin Agricultural University

Chunyu Sun

Jilin Agricultural University

Kangyu Wang

Jilin Agricultural University

Yanfang Wang

Jilin Agricultural University

Mingzhu Zhao

Jilin Agricultural University

Ping Chen

Jilin Agricultural University

Jun Lei

Jilin Agricultural University

Yi Wang

Jilin Agricultural University

Meiping Zhang

Jilin Agricultural University

Research article

Keywords: Panax ginseng, bZIP transcription factor, Phylogenetic analysis, Functional differentiation, Drought stress

Posted Date: January 3rd, 2020 
DOI: https://doi.org/10.21203/rs.2.20019/v1

License: (c) (1) This work is licensed under a Creative Commons Attribution 4.0 International License. Read Full License

Version of Record: A version of this preprint was published at BMC Genomics on May 1st, 2021. See the published version at https://doi.org/10.1186/s12864-021-07624-z. 
1 Basic Leucine zipper (bZIP) transcription factor genes and

2 their response to drought stress in ginseng, Panax ginseng

\section{C.A. Meyer}

4 Hongjie $\mathrm{Li}^{1 \llbracket}$, Jing Chen ${ }^{1,2 \pi}$, Qi Zhao ${ }^{1}$, Yilai $\operatorname{Han}^{1}$, Chunyu Sun ${ }^{1,2}$, Kangyu Wang ${ }^{1,2}$,

5 Yanfang Wang ${ }^{2,3}$, Mingzhu Zhao ${ }^{1,2}$, Ping Chen $^{1}$, Jun Lei $^{1}$, Yi Wang ${ }^{1,2^{*}}$, and Meiping

$6 \quad$ Zhang $^{1,2^{*}}$

7

$8{ }^{1}$ College of Life Science, Jilin Agricultural University, 2888 Xincheng Street, 9 Changchun, Jilin 130118, China.

$10{ }^{2}$ Research Center Ginseng Genetic Resources Development and Utilization, 2888 11 Xincheng Street, Changchun, Jilin 130118, China.

$12{ }^{3}$ College of Chinese Medicinal Materials, Jilin Agricultural University, 2888 13 Xincheng Street, Changchun 130118, Jilin, China.

*Correspondence:

E-mail: meiping.zhang@jlau.edu.cn (MPZ); wanglaoshi0606@163.com (YW)

17 IThese authors contributed equally to this work. 
19

\section{Abstract}

Background: As a famous and important medicinal herb in the world, ginseng contains numerous bioactive components that are remarkable for mankind's health. The basic leucine zipper (bZIP) transcription factor genes play important roles in many biological processes and plant response to abiotic and biotic stresses. Nevertheless, these genes remain unknown in ginseng.

Results: Here, we report 91 bZIP genes, designated as $P g b Z I P$ genes, identified from ginseng. These PgbZIP genes were alternatively spliced into 273 transcripts. Phylogenetic analysis grouped the PgbZIP genes into ten groups, including A, B, C, D, E, F, G, H, I and S. Gene Ontology (GO) categorized the PgbZIP genes into a number of functional categories, suggesting that they have substantially diversified in functionality, even though their putative proteins share a number of conserved motifs. These 273 PgbZIP transcripts expressed quite differentially across 14 different tissues, the roots of different aged, and the roots of different cultivars. However, the expression of these transcripts was coordinated as they formed a co-expression network. Furthermore, we studied their response to drought stress in ginseng using five representatives of the PgbZIP genes, including PgbZIP25, PgbZIP38, PgbZIP39, PgbZIP53 and PgbZIP54. The results showed that these PgbZIP genes all responded to drought stress in ginseng, but the magnitudes of their response to drought stress varied.

Conclusions: These results provide knowledge and resources for deeper functional analysis of PgbZIP genes and molecular tools for enhanced drought tolerance 
41

42

43

44

45

breeding in ginseng.

Keywords: Panax ginseng, bZIP transcription factor, Phylogenetic analysis, Functional differentiation, Drought stress

\section{Background}

As one member of the order Apiales in Araliaceae, ginseng (Panax ginseng C.A. Meyer) is an important medicinal herb in East Asia. In China, the cultivation of ginseng has a long history and was mainly cultivated in Jilin Province, which was known as Jilin ginseng. Ginsenosides, presenting in most tissues of ginseng, are recognized as the most valuable active components in ginseng [1, 2]. Research showed that ginseng has brought a lot of benefits for human, such as improving relieving pain, brain function, and antitumor activity, etc. [3-5]. However, ginseng is a perennial herb which suffers from different biotic or abiotic stresses frequently. Therefore, the ginseng production is greatly threatened by these challenges. To surmount such difficulties, it is urgent to analyze and utilize the defense-related genes in ginseng.

Various transcription factors (TFs) have received more and more attention in plant defense as they play a vital role in various biotic or abiotic stresses resistance. Among the TFs families, the basic leucine zipper (bZIP), contained a conserved bZIP domain composed of 60-80 amino acids, was known as one of the most conserved and largest families [6, 7]. The conserved bZIP domain was composed by two important functional regions, i.e., one basic region and one leucine zipper, linked by one hinge 
region $[8,9]$. The basic region usually contained an invariant $\mathrm{N}-\mathrm{x} 7-\mathrm{R} / \mathrm{K}$ motif (approximately sixteen amino acid), which is responsible for both nuclear localization and DNA binding. In contrast, the leucine zipper regions mediate the homo- and/or heterodimerization as they contained a less conserved dimerization motif [10-13].

Considerable evidence demonstrated that many bZIP genes play a vital role in various biological processes, including the differentiation of many tissues and organs, vascular development, initiation in plants [14, 15], embryogenesis [16], and seed maturation [17]. However, there is increasing evidence has indicated that bZIP protein can also be used as key components in plant regulation of biotic and abiotic stresses, e.g., pathogen defense [18, 19], osmosis [20, 21], high salt environment [22, 23], cold $[13,24]$, and drought $[25,26]$. For example, it has been reported that AtbZIP28 was activated by thermal impact, and then the expression of heat-responsive genes increased to protect the plants from heat [27]. In rice, bZIP23 and bZIP72 were reported to play a critical role in attenuating drought treatment by activating $\mathrm{ABA}$ signaling $[28,29]$. Knockout slbZIP1 genes and slAREB1, belonging to subfamily A of bZIP TF, increased salt stress tolerance, while overexpression of slbZIP1 and slAREB1 decreased salt stress tolerance in Solanum lycopersicum [30, 31].

The bZIP TF families have been analyzed in various plant species, varying among plants. For example, there were 75 bZIP genes were identified in Arabidopsis [10], 69 genes in tomato [32] and 89 members in rice [7]. However, there is currently no research on the bZIP family in ginseng. Herein, the bZIP genes from ginseng were identified, and then the phylogenetic analysis, conserved protein motifs, and predicts 
functional analysis, as well as expression characteristics were performed. Due to drought stress is an important factor for astricting the growth and development of plants [33], the expression level of five bZIP genes in drought stress was also quantized by quantitative real-time PCR (qRT-PCR) to verify their role in drought resisting.

\section{Materials and methods}

\section{Database resources}

The Jilin ginseng transcriptome database developed from 14 different tissues of Jilin ginseng of four years and the roots of different years old were both used in this study [34]. In addition, the transcriptomes derived from the four-year-old plants root of 42 different farmers' cultivars of ginseng in Jilin province, China (named from S1 to S42) $[35,36]$ were also consulted.

\section{Genetic differences of ginseng in different regions}

In this study, we first downloaded the sequence of bZIP transcription factor from Ginseng Genome Database (http://ginsengdb.snu.ac.kr/index.php) [37]. Above bZIP transcription factor sequences were aligned with bZIP transcription factor identified from Jilin ginseng transcriptome database. The sequences with identity $\geqslant 95 \%$, alignment length $\geqslant 240$ bp (bZIP maximum domain length) were identified as the same gene in this study.

\section{Identification and phylogenetic analyses of $b Z I P$ gene family} in ginseng 
105

106

107

108

109

110

111

112

113

114

115

116

117

118

119

120

121

122

123

124

125

Firstly, the local Hidden Markov Model (HMM) search was carried out on the basis of

HMM profile of the bZIP domain (including PF07716, PF00170, PF03131 and PF012498) (http://pfam.sanger.ac.uk/) using HMMER3.0 (HMMER: http://hmmer.wustl.edu/). Then, BLAST searches were carried out with a threshold of 1.0E-06 to authenticate the predicted $b Z I P$ genes in ginseng transcriptome database. Then, all the candidate nucleic acid sequences were filtered by the Online Conserved Domains (CDS) search and the resulting genes were identified as PgbZIP gene.

The whole bZIP protein sequences from Arabidopsis thaliana (Arabidopsis), Solanum lycopersicum (tomato) and Oryza sativa (rice) were obtained from the plant TF database (PlantTFDB, v3.0) [38],k Sol Genomics Network (SGN; http://solgenomics.net/) and Rice Genome Annotation Project RGAP (http://rice.plantbiology.msu.edu/) databases [32], respectively. Then, all these full proteins encoded by bZIP genes of Arabidopsis, rice, tomato and ginseng were subjected to muscle to perform multiple sequence alignments. Finally, the $\begin{array}{lllllll}\text { phylogenetic tree was constructed using } & \text { MEGA version } & 7.0\end{array}$ (http://www.megasoftware.net), and the parameter is the neighbor-joining (NJ) algorithm, 1000 replicates, pairwise deletion of gaps and p-distance method.

\section{Conserved motifs analyses}

The full length of the PgbZIP transcripts were subjected to the online ORF Finder at NCBI (http: //www. ncbi.nlm.nih.gov/gorf/gorf.html) to search their open reading frames (ORFs). Then, the conserved motifs of the PgbZIP protein were searched 
using the Motif

Elicitation

tool

(version

4.9.1,

127

http://meme.sdsc.edu/meme/cgi-bin/meme.cgi) [39] with a maximum number of 20 .

The minimum width, maximum width of the conserved motifs was 10 and 50, respectively and other parameters were used as default [40].

\section{Functional categorization of the PgbZIP transcripts}

The 273 PgbZIP transcripts were submitted to Blast2GO software (Version 4.1.5) to perform GO analysis. After predict the functional differentiation of PgbZIP transcripts, the enrichment of these functional subcategories were analyzed [41]. In the enrichment analysis, the GO function classification of 273 unknown ginseng gene transcripts randomly selected throughout the Jilin ginseng transcriptome database was used as a control (level 2). The significance test between the number of PgbZIP transcripts and 273 unknown ginseng transcripts classified into each functional subcategory were determined by the Chi-square test.

\section{Expression and network analysis of the PgbZIP transcripts}

The expression of 273 PgbZIP transcripts was extracted from the following transcriptome databases: the 14 tissues in one four-year-old ginseng, the roots of 5-, 12-, 18- and 25-year-old ginseng [34], as well as the four-year-old roots of 42 ginseng genotypes in Jilin, China [35]. We used the R programming language and software to construct the expression heatmaps of the 273 PgbZIP transcripts, and the BioLayout Express3D software (Version 3.2) to construct the gene coexpression networks [42].

\section{Plant growth and stress treatments}


The ginseng seeds were obtained from Jilin Province, China, and were grown in the pots containing nutritional soil for 4 weeks. Then, the four-week-old ginseng seedlings were treated by $20 \%$ PEG-6000 to simulate the drought stress treatments. After treated with PEG-6000 for $3 \mathrm{~h}, 6 \mathrm{~h}, 12 \mathrm{~h} 24 \mathrm{~h}$ and $48 \mathrm{~h}$, the entire ginseng seedlings were harvested and weighed straightway (fresh weight) at indicated time points. The seedlings were placed in a vial containing $4{ }^{\circ} \mathrm{C}$ distilled water for $24 \mathrm{~h}$, and the saturated weight was measured. Then, these seedlings dried at $70{ }^{\circ} \mathrm{C}$ for $72 \mathrm{~h}$, and the dry weight was measured. Finally, the relative water content (RWC) of the control and drought stress treated seedlings was measured using the following formula: [(fresh weight - dry weight) / (saturated weight - dry weight)] x 100 [43].

\section{RNA isolation and quantitative real-time PCR analysis}

After treat with PEG -6000 for $3 \mathrm{~h}, 6 \mathrm{~h}, 12 \mathrm{~h} 24 \mathrm{~h}$ and $48 \mathrm{~h}$, the total RNA of ginseng seedlings were isolated by TRIzol reagent (Biotake, Beijing, China) following the manufacturer's instructions and the first-strand cDNAs were also synthesized by the PrimeScript $^{\mathrm{TM}}$ RT reagent Kit with gDNA Eraser (TaKaRa, Tokyo, Japan) according to the manufacturer's instructions. Ubiquinol-cytochrome $\mathrm{C}$ reductase gene (QCR) of ginseng was selected as marker genes for drought treatments [44]. For the PgbZIP genes, the specific primers were also designed and synthesized and the primers were listed in Additional file 1: Table S1. qRT-PCR was conducted using an Applied Biosystems 7500 Real Time PCR System (Thermo Fisher Scientific, Waltham, MA, USA) and SYBR Premix Ex Taq ${ }^{\mathrm{TM}}$ II (TaKaRa, Tokyo, Japan). The PCR conditions were $30 \mathrm{~s}$ at $95{ }^{\circ} \mathrm{C}$, and followed by forty cycles of $5 \mathrm{~s}$ at $95{ }^{\circ} \mathrm{C}, 34 \mathrm{~s}$ at $60{ }^{\circ} \mathrm{C}$, and 
170 replicates were set and the genes relative expression was calculated using the $2^{-\Delta \Delta \mathrm{Ct}}$ 171 formula.

\section{Results}

\section{Identification of PgbZIP genes}

After searching from the 248,993 unigenes in the ginseng transcriptome database created in our previous study, 1957 gene sequences were characterized from ginseng. However, as the CDS of 1684 gene sequences were incomplete or outside the ORFs, the left 273 transcripts containing complete bZIP domain inside their ORFs were identified from Jilin ginseng. These 273 transcripts had a sequence of 210 to $3,651 \mathrm{bp}$, with an average number of 1449 bp (Additional file 2: Table S2). By Trinity software, these 273 transcripts were defined to derive from 91 bZIP genes, which were further named PgbZIP 01 to 91 [45]. The multiple transcripts derived from one $P g b Z I P$ gene were suffixed with another Arabic number, e.g., PgbZIP11-1 and PgbZIP11-2. Among the 273 PgbZIP transcripts, 190 contained full-length open-reading frames (ORF), which were derived from 62 PgbZIP genes (Additional file 3: Table S3). The encoded full-length proteins by 190 PgbZIP gene transcripts contained amino acids varying from 46 (PgbZIP84) to 785 (PgbZIP63-1), with an average of 294. Comparing to the sequences of Korean ginseng bZIP from Ginseng Genome Database (http://ginsengdb.snu.ac.kr/index.php), 45 (49\%) Jilin ginseng bZIP genes identified in this study were similar $(\geqslant 95 \%)$ to $111(76 \%)$ Korean ginseng bZIP, which could 
190

191

192

193

194

195

196

197

198

199

200

201

202

203

204

205

206

207

208

209

210

be supposed to the same genes (Additional file 4: Table S4). However, 46 (51\%)

Jilin ginseng bZIP genes identified in this study were assigned as newly discovered bZIP genes, as their sequences were quite different from Korean ginseng bZIP from Ginseng Genome Database (Additional file 5: Figure S1).

\section{Motif identification and phylogeny of the PgbZIP gene family}

To study the structural characteristics of PgbZIPs, 20 conserved motifs of PgbZIP proteins encoded by 62 PgbZIP genes were predicted using online MEME software. The distribution of each motif was shown in Fig. 1a. Motif 1, which was annotated as bZIP domain, presented in all PgbZIP members. Besides, most PgbZIP proteins of one subfamily usually exhibited similar motif features (Fig. 1a). For instance, most PgbZIP members of subfamily A contained collective motifs of 12, 15, 16 and 17; subfamily D harbors the same motifs of 2, 4, 6, 7, 8, 13 and 20; subfamily G shares motif 11. Additionally, multiple subfamilies can share the same motif. For instance, subfamily $\mathrm{G}$ and $\mathrm{S}$ possess motifs 10; and motifs 14 appeared in subfamily $\mathrm{F}$ and $\mathrm{G}$. These results indicated that PgbZIP TFs in the same groups showed similar motif characteristic, suggesting their functional similarities.

Then, the longest transcript of the 62 genes containing full-length ORF was used as a representative to construct the phylogenetic tree. Fifty six $b Z I P$ genes, which were identified from Arabidopsis, rice and tomato (Additional file 6: Table S5), were obtained from a previous study and constructed phylogenetic tree together with the 
PgbZIP gene. As shown in Fig. 1b, these 62 PgbZIPs could be clustered into ten clades, together with the outgroup bZIPs from Arabidopsis, tomato and rice. The ginseng PgbZIP family still has same number of clades with Arabidopsis, rice, tomato and maize [8], but contains more clades than castor bean [46], cucumber [47] and sorghum [48]. Comparing with these outgroup species, groups A contained 11 members in Jilin ginseng, possessing the biggest subfamily in ginseng, whereas subfamily $\mathrm{H}$ only have one gene (PgbZIP13), suggesting the function differentiation among different members of PgbZIP gene family.

\section{Functional differentiation of the PgbZIP gene family}

The functional differentiation of these 273 transcripts derived from $91 P g b Z I P$ genes were functionally categorized by the in silico Gene Ontology (GO) terms. 251 PgbZIP transcripts were annotated into different functional categories (Fig. 2 and Additional file 7: Table S6). Specifically, 235 PgbZIP transcripts were categorized to BP, while 249 PgbZIP transcripts has functions in MF and only 2 PgbZIP transcripts were annotated to cellular CC, respectively (Fig. 2a). At Level 2, the 251 transcripts were categorized into 5 subcategories, including two BP subcategories: transcription, DNA-templated and regulation of gene expression; two MF subcategories: nucleic acid binding transcription factor activity and DNA binding; and one CC subcategories: cytosol (Fig. 2b). Furthermore, among these 5 subcategories, the 4 subcategories except cytosol were significantly enriched relative to the 273 unknown ginseng transcripts randomly selected throughout the transcriptome database $(\mathrm{P} \leq 0.01)$. 
The functions of the PgbZIP gene transcripts expressed in the roots of 4 differently aged plants (Fig. 3a), 14 tissues (Fig. 3b) and 42 farmers' cultivars (Fig. 3c), were also categorized respectively. Generally, these transcripts were also categorized into the above mentioned 5 subcategories, but the numbers of the PgbZIP gene transcripts in these 5 subcategories (Level 2) varied greatly among the different age, tissues and cultivars. The above results also demonstrated the potential functional diversity of these PgbZIP superfamily genes but also confirmed their functional consistency among different developmental stages, tissues and genotypes.

\section{Expression characteristics of the PgbZIP gene transcripts in}

\section{ginseng}

To understand the possible roles of PgbZIP genes, the expression profiles of the 273 PgbZIP transcripts in different-aged plants, 14 different tissues, and 42 different farmers' cultivars were investigated (Additional file 8: Table S7). The expression profile was analyzed and the expression heatmap were constructed using PgbZIP transcripts because different transcripts of one gene probably different biological functions [49]. The expression profile of PgbZIP transcript varied dramatically among different-aged plant roots, different tissues, and different cultivars, ranging from silent to 178.53 TPM, 307.66 TPM and 169.85 TPM, respectively.

Specifically, 248 PgbZIP transcripts expressed in at least one tissue, and most PgbZIP genes usually expressed in two or more tissues of Jilin ginseng (Fig. 4a, Fig. 5 and Additional file 9: Figure S2a). Among the 248 expressed PgbZIP transcripts, only 68 
PgbZIP transcripts, i.e., 25\%, expressed in all tissues. Besides, 37 PgbZIP gene transcripts, accounting for $14 \%$, showed tissue-specific expression as they specifically expressed in one tissue. On the other hand, a total of 208 PgbZIP transcripts were found to express in at least one farmer's cultivars in Jilin province (Fig. 4b). As for each farmer's cultivars, the numbers of the expressed transcripts varied from 106 to 150, which were relatively consistent (Additional file 9: Figure S2b). Finally, 167 PgbZIP transcripts expressed at least in one of 5-, 12-, 18- and 25-year-old ginsengs roots (Fig. 4c), while 131, 120, 125 and 130 PgbZIP transcripts were separately expressed in above four years-old roots (Additional file 9: Figure S2c), which were also relatively consistent. To analyze the expression pattern of ginseng bZIP, we constructed a heat map based on the ginseng transcriptome database. Fig. 5 showed the expression heatmap of the PgbZIP gene superfamily in different tissues, different cultivars, and different years-old ginsengs. Generally, the expression of PgbZIP transcripts varied greatly among different tissues, cultivars, and developmental stages.

\section{The network of PgbZIP genes expression}

To detect the expression association among different members of the PgbZIP genes, the co-expression networks of 273 PgbZIP transcripts were constructed basing on their expression profile in 14 different tissues and 42 different farmers' cultivars, respectively, at a value of $\mathrm{P} \leq 5.0 \mathrm{E}-02$ (Additional file 10: Figure S3 and Fig. 6). Meanwhile, 273 unknown transcripts of ginseng genes were randomly extracted from the 248,993 ginseng unigenes and used for negative controls. In 14 different tissues of ginseng, the expression profile of 248 PgbZIP transcripts expressed in at least one 
tissues formed a co-expression network with 248 nodes, 2,429 edges (Additional file 10: Figure S3a), and 20 clusters (Additional file 10: Figure S3b, Additional file 11: Table S8). Comparing with the negative control, PgbZIP transcripts contained fewer edges. However, PgbZIP transcripts possessed evidently more nodes than negative controls at P-values from 1.0E-02 to 1.0E-03 (Additional file 10: Figure S3c-f). In the 42 different farmers' cultivars, 208 PgbZIP transcripts tended to expressed connectedly and form a co-expression network composed of 207 nodes, 1994 edges (Fig. 6a), and 18 clusters (Fig. 6b, Additional file 12: Table S9). Statistical analysis showed that these PgbZIP transcripts were tended to form a closer co-expression network much farther than the randomly extracted transcripts in 42 ginseng cultivars (Fig. 6c-f).

\section{PgbZIP genes actively respond to drought}

To further speculate the function of $P g b Z I P$ genes in drought stresses response, the expression patterns of 5 PgbZIPs randomly selected from several large subfamilies, i.e. PgbZIP25, PgbZIP38, PgbZIP39, PgbZIP53 and PgbZIP54, were analyzed by q-RT PCR. To simulate drought stresses, ginseng seedlings were exposed with $20 \%$ (w/v) PEG-6000, for 3, 6, 12, 24 and $48 \mathrm{~h}$ and then the RWC were determinated. As a result, the RWC of seedlings treated with PEG-6000 for $6 \mathrm{~h}$ were significantly reduced comparing with the control plants. However, there was no significant difference in RWC at other time points (Additional file 13: Figure S4). On the other hand, the expressions of these five PgbZIP genes in the ginseng seedlings were mainly up-regulated by PEG-6000 treatment. Specifically, the expression of 
PgbZIP25, PgbZIP38, PgbZIP39, PgbZIP53 and PgbZIP54 genes treated with PEG-6000 for $3 \mathrm{~h}$ were significantly up-regulated by 4.11, 12.62, 8.62, 4.74 and 18.93 times comparing to the untreated plants $(\mathrm{P}<0.01)$. However, with time going on, the expression patterns of these five genes are different from each other. For instance, the expression of PgbZIP25 reached to the crest after drought stress treated for $6 \mathrm{~h}$ while showed no significant difference with the control plants after treated for $12 \mathrm{~h}, 24 \mathrm{~h}$ and 48 h. PgbZIP38 gene was continuously up-regulated by PEG-6000 treated for 3h-24h but returned to normal level at $48 \mathrm{~h}$. Noteworthy, except $3 \mathrm{~h}$, the expression of PgbZIP53 in ginseng seedling exposed with PEG-6000 for other time points showed no significant difference with the control. On the other hand, the left two genes, i.e. PgbZIP39 and PgbZIP54, showed irregular variation (Fig. 7).

\section{Discussion}

To date, due to the signicant role in regulate various biological processes, the bZIP TFs has been attractive and identified in various plants, e.g. Arabidopsis (75 members) [10], maize (125 genes) [8], soybean (160 genes ) [13], grapevine (55 genes) [50], castor bean (49 members) [46], cucumber (64 members ) [47], sorghum (92 genes) [48], barley (89 genes) [12] and Brachypodium distachyon (96 members) [9], respectively. Herein, we have identified 91 bZIP genes from Jilin ginseng. Comparing with the members of bZIP in other plants, the number of bZIP gene family in ginseng was similar to that in rice, barley, sorghum and Brachypodium distachyon, but expanding to Arabidopsis, castor bean (49), and cucumber (64), and grapevine (55), whereas shrunk to maize (125) and soybean (160). 
Phylogenetic tree showed that the PgbZIPs could be divided into 10 subfamilies, which has the same evolutionary classification as bZIPs of Arabidopsis, rice, tomato and maize, suggesting similar evolutionary trajectories between ginseng and these plants (Fig. 1b). Notably, most clades contained both bZIPs of ginseng, Arabidopsis, tomato and rice, suggesting that at least some members of the bZIPs have been existed before the differentiation of monocots and dicots. The interspecies clustering also suggested that some homologous $b Z I P$ genes were existed between ginseng, Arabidopsis, rice and tomato. Therefore, by phylogenetic analyses, bZIPs were supposed to have a relatively conservative structure and function among different plant species when evolution. Additionally, conserved motif analysis revealed that the typical bZIP domain was found in almost all the PgbZIPs and different subfamilies usually had some distinguishable motifs. However, sometimes some groups shared the same special motifs (Fig. 1a), which were consistent with the features of bZIPs in grapevine [50].

Numerous bZIP proteins were reported to involve in various biological processes, e.g., the drought/osmotic stress response [9, 47, 48], growth and development, cell elongation [51, 52], organ and tissue differentiation [53] and the gene regulation of seed storage protein [17]. By GO terms in silico, PgbZIPs were functionally classified into 5 subcategories, including transcription, DNA-templated, regulation of gene expression, nucleic acid binding transcription factor activity, DNA binding and cytosol. However, the number of the functional differentiation of bZIPs gene family was a lot less than the $R L K$ gene family (23 subcategories) [54] and $N B S$ gene family 
341 (36 subcategories) [35] in ginseng. Our results indicate that the encoded bZIP proteins

342 in ginseng might mainly worked as TFs by binding to the promoter region of specific

343 genes and different members probably perform similar functions in some specific

344 biological processes.

345 Furthermore, transcriptional patterns of PgbZIP transcripts were analyzed using three

346 transcriptome databases [34] of Jilin ginseng reported previously. The heatmap based

347 on three transcriptome databases suggested that most genes expressed in seeds, roots,

348 stems, leaves and flowers, which was in consistent with the studies in Arabidopsis

349 [10]. However, these PgbZIP genes exhibited significant variations in expression

350 across different tissues, also similar to the other plants, e.g., studies in rice and maize

351 [7, 8]. Sometimes, different transcripts of the same gene exhibited different

352 expression patterns, which might be resulted from the alternatively splicing of 353 pre-RNA.

354 Unexpectedly, despite of the structure, functional differentiation and expression 355 activity among PgbZIP transcripts exhibited a noteworthy diversity, the majority of the PgbZIP transcripts remained to correlatively express and form a co-expression

357 network by analyzing their expression association. Besides, the networks among

PgbZIP transcripts was markedly stronger than that in unknown the transcripts

359 randomly extracted form ginseng transcriptome databases, suggesting that PgbZIP transcripts probably somehow interact with each other or remain functionally coordinated (Additional file 13: Figure S4). On the other side, the numerous clusters 
differentiation among different $P g b Z I P$ transcripts.

As a perennial herb, ginseng has been frequently suffering from various environmental stresses. However, to date, the mechanisms of the resistance of ginseng to environmental stress were not clearly clarified. Herein, to excavate potential $b Z I P$ genes resisting to the drought stress in ginseng, the PEG were selected to simulate drought treatment and exposed to ginseng as it was reported that PEG could significantly change the relative water content in plants [43]. It was reported that the group A of PgbZIPs play a significant role in the drought and high salinity stress [55-57]. e.g., overexpression of OsbZIP46, a member of group A, could significantly improve both drought resistance and osmotic resistance in rice [58]; ThbZIPI gene of Tamarix hispida, can increase the tolerance to salt and drought stress in Arabidopsis [59]. Herein, the expression level of five randomly selected PgbZIP genes, including a group A, i.e., PgbZIP39, under drought stresses were analyzed by qPCR. As a result, PgbZIP39 was significantly up-regulated (8.62 times comparing to the untreated plants) by PEG-6000 treated. Besides, the other four genes, (PgbZIP25, PgbZIP38, PgbZIP53 and PgbZIP54 genes), randomly selected from the S, G, S and I groups, also showed significantly transcriptional changes $(\mathrm{p}<0.01)$ after drought stress treatments at least one time point. Therefore, it can be speculated that besides group A, other groups of $P g b Z I P$ genes, probably also play an essential role in responding drought stresses. The above results could provide valuable information for the functional verification of $P g b Z I P$ genes and identifying new drought resistance genes in ginseng. However, it is still far from unraveling the exact biological functions of 
PgbZIPs, which needs to be further investigated.

\section{Conclusions}

387

91 bZIP genes were identified from ginseng and systematically analyzed by phylogenetic analyses, conserved motif and functionally categorizing in silico. Besides, the expression characteristics and network were also analyzed. The function of PgbZIP genes were obviously differentiated, the expression of PgbZIP genes are also varied in spatial and temporal in ginseng. Simultaneously, under drought stress treatments, the PgbZIP genes expression levels suggested that several groups of PgbZIP were involving in drought signaling. Together, this study showed some new characteristics of the bZIP TFs gene family in Jilin ginseng, which would not only provided some basis for further understanding the evolution, expression and functional prediction of the PgbZIPs, but also increased our understanding the role of PgbZIP genes in the regulation of drought resistance in ginseng.

\section{Figure Captions}

Fig. 1 Conserved motifs and Phylogenetic relationship of the PgbZIP proteins. a Conserved motifs of PgbZIP proteins according to the evolutionary relationship. The conserved motifs in the PgbZIP proteins were identified with MEME software. Each motif is indicated by a colored box numbered at the top. b Phylogenetic analysis of ginseng, Arabidopsis, tomato and rice bZIP proteins.

Fig. 2 In silico functional categorization and GO term enrichment of the PgbZIP gene transcripts. a Venn diagram of numbers of the PgbZIP transcripts involved in the biological process (BP) (235 genes), molecular function (MF) (249 
genes)and cellular component (CC) (2 genes) categories. b Subcategories in which the PgbZIP transcripts are involved at Level 2 and their enrichments. The GO terms of the transcripts expressed in 14 tissues of the four-year-old plant used for identification of the PgbZIP genes as the background control for the enrichment analysis. ${ }^{*}$, significant at $\mathrm{P} \leq 0.01$; the remaining GO terms are not significant at $\mathrm{P} \leq 0.05$.

Fig. 3 Variation of the functional categories of the PgbZIP transcripts. a Variation of the functional categories of the PgbZIP transcripts among 14 tissues of a 4-year-old plant. b Variation of the functional categories of the PgbZIP transcripts among the 4-year-old roots of 42 ginseng cultivars from Jilin, China. c Variation of the functional categories of the PgbZIP transcripts among the roots of differently aged plants.

Fig. 4 Expression of the PgbZIP gene transcripts in ginseng. a Expression of the PgbZIP gene transcripts in different numbers of tissues. The percentages of each part of the pie indicates the percentage of the 273 PgbZIP gene transcripts; the number behind the percentage in each part of the pie indicates the number of the 14 tissues in which the gene transcripts expressed. b Expression of the PgbZIP gene transcripts in four-year-old roots of different genotypes. The percentages of each part of the pie indicates the percentage of the 273 PgbZIP gene transcripts; the number behind the percentage in each part of the pie indicates the number of the four-year-old roots of different genotypes in which the gene transcripts expressed. c Expression of the PgbZIP gene transcripts in differently-aged roots.. The percentages of each part of the pie indicates the percentage of the 273 PgbZIP gene transcripts; the number behind the percentage in each part of the pie indicates the number of the 
number of four aged roots in which the gene transcripts expressed.

434

Fig. 5 Expression of the PgbZIP transcripts categorized into the immune system process. a Expression of the PgbZIP transcripts in 14 tissues. b Expression of the PgbZIP transcripts in 42 cultivars. c Expression of the PgbZIP transcripts in four different year-old roots.

Fig. 6 Network analysis of the PgbZIP transcripts expressed in the 4-year-old roots of 42 farmers' cultivars. a The co-expression network constructed from 273 PgbZIP transcripts at $\mathrm{P} \leq 5.0 \mathrm{E}-02$. It consists of 207 nodes and 1994 edges. b 18 clusters in the network. c Tendency that PgbZIP genes form a network using the randomly-selected ginseng unknown genes as a control: variation in number of nodes. d Tendency that PgbZIP genes form a network using the randomly-selected ginseng unknown genes as a control: variation in number of edges. The networks shown in (c and d) were constructed from 273 transcripts with no replicate. e Statistics of variation in number of nodes in the PgbZIP network. f Statistics of variation in number of edges in the PgbZIP network. Capital letters, significant at $\mathrm{P} \leq 0.01$; Error bar, the standard deviation for 20 bootstrap replications.

Fig. 7 PgbZIP gene expression levels in ginsen seedlings after 3, 6, 12, 24 and $48 \mathrm{~h}$ of 20\% PEG-6000 treatment. The values are expressed as the means of 3 replicates. ${ }^{*}: \mathrm{P}<0.05 . * *: \mathrm{P}<0.01$.

\section{Additional files}

Additional file 1: Table S1. Primers used in qRT-PCR analysis. (XLSX 57 kb)

Additional file 2: Table S2. The PgbZIP genes identified in this study and their transcript sequences. (XLSX $132 \mathrm{~kb}$ ) 
Additional file 3: Table S3. The PgbZIP genes and their putative protein sequences. (XLSX $30 \mathrm{~kb}$ )

Additional file 4: Table S4. Sequence Alignment between bZIP transcription factor from Ginseng Genome Database and bZIP transcription factor identified from Jilin ginseng transcriptome database. (XLSX $13 \mathrm{~kb}$ )

Additional file 5: Figure S1. Comparison of bZIP transcription factor from Ginseng Genome Database and bZIP transcription factor identified from Jilin ginseng transcriptome database. (TIFF $4471 \mathrm{~kb})$

Additional file 6: Table S5. The published bZIP genes used as outgroup for classification and phylogenetic analysis of the PgbZIP gene superfamily. (XLSX 47 $\mathrm{kb})$

Additional file 7: Table S6. Annotation and GO categorization of the 273 PgbZIP transcripts. (XLSX $25 \mathrm{~kb}$ )

Additional file 8: Table S7. Expressions of the PgbZIP transcripts in the four-year-old roots of 42 farmers' cultivars, 14 tissues of a four-year-old plant and 4 different year-old roots (TPM). (XLSX $97 \mathrm{~kb}$ )

Additional file 9: Figure S2. Expression of the PgbZIP transcripts in ginseng. a Number of cultivars in the four-year-old roots of which the PgbZIP transcripts expressed. b Number of tissues in which the PgbZIP transcripts expressed. c Venn diagram of numbers of the PgbZIP transcripts expressed in differently-aged roots. (TIFF $854 \mathrm{~kb})$

Additional file 10: Figure S3. Network of the PgbZIP transcripts expressed in 14 tissues of a four-year-old ginseng plant. a The co-expression network constructed 
from 440 PgbZIP transcripts at $\mathrm{P} \leq 5.0 \mathrm{E}-02$. It consists of 248 nodes and 2429 edges. b 20 clusters in the network. c Tendency that PgbZIP genes form a network using the randomly-selected ginseng unknown genes as a control: variation in number of nodes. d Tendency that PgbZIP genes form a network using the randomly-selected ginseng unknown genes as a control: variation in number of edges. The networks shown in (c and d) were constructed from 273 transcripts with no replicate. e Statistics of variation in number of nodes in the PgbZIP network. f Statistics of variation in number of edges in the PgbZIP network. Capital letters, significant at $\mathrm{P} \leq 0.01$; Error bar, the standard deviation for 20 bootstrap replications. (TIFF $3663 \mathrm{~kb}$ )

Additional file 11: Table S8. Matrix of expression correlation coefficients between the PgbZIP gene transcripts in 14 tissues of a fouryearold plant used for coexpression network construction of the PgbZIP genes in different tissues. (XLSX $673 \mathrm{~kb}$ )

Additional file 12: Table S9. Matrix of expression correlation coefficients between the PgbZIP gene transcripts in the roots of fouryearold plants of 42 cultivars used for coexpression network construction of the PgbZIP genes in the roots of different cultivars. (XLSX $606 \mathrm{~kb}$ )

Additional file 13: Figure S4. Relative water content of ginseng seedlings subjected to PEG stress. The control plants were maintained under normal water irrigation conditions. The values are expressed as the means of 3 replicates. *: $\mathrm{P}<0.05$. **: $\mathrm{P}<$ 0.01. (TIFF $295 \mathrm{~kb}$ )

\section{Abbreviations}

Ginseng: Panax ginseng C.A. Meyer; bZIP: basic leucine zipper; PgbZIP: Panax ginseng bZIP; ORF: Open reading frame; CDS: Conserved Domains; MEME: Multiple EM for motif elicitation; GO: gene ontology; MF: molecular function; BP: 
biological process; CC: cellular component; HMM: Hidden Markov Model; RWC:

Relative water content

\section{Ethics approval and consent to participate}

Not applicable

\section{Consent for publication}

Not applicable

\section{Availability of data and materials}

Not applicable

\section{Competing interests}

The authors declare that the research was conducted in the absence of any commercial or financial relationships that could be construed as a potential conflict of interest.

\section{Funding}

Not applicable

\section{Authors' contributions}

MPZ and YW planned and designed this study; HL performed the work and wrote the manuscript; HL, QZ and YH performed drought stress and conducted qRT-PCR; CS, KW, YFW, PC and JL prepared the tables and figures. JC and MPZ revised the manuscript. All the authors read and approved the final version of the manuscript.

\section{Acknowledgements}


523

524

525

526

527

528

529

530

531

532

533

534

535

536

537

538

539

540

541

542

543

544

This research was supported by an award from China 863 Project (2013AA102604-3),

the Bureau of Science and Technology of Jilin Province (20190201264JC,

20170101010JC, 20180414077GH, 20180101027JC), the Development and Reform

Commission of Jilin Province (2016C064, 2018C047-3), and a startup fund from Jilin

Agriculture University (201801, https://www.jlau.edu.cn/).

\section{Author details}

${ }^{1}$ College of Life Science, Jilin Agricultural University, 2888 Xincheng Street, Changchun, Jilin 130118, China. ${ }^{2}$ Research Center Ginseng Genetic Resources Development and Utilization, 2888 Xincheng Street, Changchun, Jilin 130118, China. ${ }^{3}$ College of Chinese Medicinal Materials, Jilin Agricultural University, 2888 Xincheng Street, Changchun 130118, Jilin, China.

\section{References}

1. Choi KT. Botanical characteristics, pharmacological effects and medicinal components of Korean Panax ginseng C A Meyer. Acta Pharmacolo Sin. 2008; 29(9), 1109-1118.

2. Zhang YC, Li G, Jiang C, Yang B, Yang HJ, Xu HY, Huang LQ. Tissue-specific distribution of ginsenosides in different aged ginseng and antioxidant activity of ginseng leaf. Molecules. 2014; 19(11), 17381-17399.

3. Popovich DG, Yeo SY, Zhang W. (2010). Ginseng (Panax quinquefolius) and Licorice (Glycyrrhiza uralensis) Root Extract Combinations Increase Hepatocarcinoma Cell (Hep-G2) Viability. Evid Based Complement Alternat Med. 2010; 2011(1), 408273.

4. Lee ST, Chu K, Sim JY, Heo JH, Kim M. Panax ginseng enhances cognitive performance in 
5. Xie X, Wang HT, Li CL, Gao XH, Ding JL, Zhao HH, Lu YL. Ginsenoside Rb1 protects PC12 cells against $\beta$-amyloid-induced cell injury. Mol Med Rep. 2010; 3(4), 635-639.

6. Alves MS, Dadalto SP, Gonçalves AB, De Souza GB, Barros VA, Fietto LG. Plant bZIP transcription factors responsive to pathogens: a review. Int J Mol Sci. 2013; 14, 7815-7828.

7. Nijhawan A, Jain M, Tyagi AK, Khurana JP. Genomic survey and gene expression analysis of the basic leucine zipper transcription factor family in rice. Plant Physiol. 2008; 146(2),

8. Wei K, Chen J, Wang Y, Chen Y, Chen S, Lin Y, Pan S, Zhong X, Xie D. Genome-wide analysis of bZIP-encoding genes in maize. DNA Res. 2012; 19(6), 463-476. factors and their expression profiles in response to multiple abiotic stresses in Brachypodium

10. Jakoby M, Weisshaar B, Drögelaser W, Vicentecarbajosa J, Tiedemann J, Kroj T, Parcy F; bZIP Research Group. bZIP transcription factors in Arabidopsis. Trends Plant Sci. 2002; 7(3),

11. Foster R, Izawa T, Chua NH. Plant bZIP proteins gather at ACGT elements. Faseb J. 1994;8(2), 192.

12. Pourabed E., Golmohamadi FG, Monfared PS, Razavi SM, Shobbar ZS. Basic Leucine Zipper 

and confer salt and freezing tolerance in transgenic Arabidopsis. Planta. 2008; 228(2), 225-240.

14. Abe M, Kobayashi Y, Yamamoto S, Daimon Y, Yamaguchi A, Ikeda Y, Ichinoki H, Notaguchi M, Goto K, Araki T. FD, a bZIP Protein Mediating Signals from the Floral Pathway Integrator FT at the Shoot Apex. Science. 2005; 309(5737), 1052-1056.

15. Silveira AB, Gauer L, Tomaz JP, Cardoso PR, Carmello-Guerreiro S, Vincentz M. The and vascular development. Plant Sci. 2007; 172(6), 1148-1156.

16. Guan Y, Ren H, Xie H, Ma Z, Chen F. Identification and characterization of bZIP-type transcription factors involved in carrot (Daucus carota L.) somatic embryogenesis. Plant J. 

dehydrogenase transcription by specific heterodimerisation of bZIP transcription factors. Embo J. 2014; 25(13), 3133-3143.

21. Balázs A, Pócsi I, Hamari Z, Leiter E, Emri T, Miskei M, Oláh J, Tóth V, Hegedus N, Prade RA, Molnár M, Pócsi I. AtfA bZIP-type transcription factor regulates oxidative and osmotic stress responses in Aspergillus nidulans. Mol Genet Genomics. 2010; 283(3), 289-303. basic leucine zipper transcription factors involved in an abscisic acid-dependent signal transduction pathway under drought and high-salinity conditions. Proc Natl Acad Sci U S A. 2000;97(21), 11632-11637.

23. Huang C, Zhou J, Jie Y, Xing H, Zhong Y, She W, Wei G, Yu W, Ma Y. A ramie (Boehmeria nivea) bZIP transcription factor BnbZIP3 positively regulates drought, salinity and heavy metal tolerance. Mol Breed. 2016; 36(8), 120.

24. Shimizu H, Sato K, Berberich T, Miyazaki A, Ozaki R, Imai R, Kusano T. LIP19, a basic region leucine zipper protein, is a fos-like molecular switch in the cold signaling of rice plants. Plant Cell Physiol. 2005; 46(10), 1623-1634.

25. Ying S, Zhang DF, Fu J, Shi YS, Song YC, Wang TY. Cloning and characterization of a maize bZIP transcription factor, ZmbZIP72, confers drought and salt tolerance in transgenic Arabidopsis. Planta. 2012; 235(2), 253-266.

26. Liu C, Wu Y, Wang X. bZIP transcription factor RISBZ5: a potential negative regulator of cold and drought stress response in rice. Planta. 2012; 235(6), 1157-1169.

27. Gao H, Brandizzi F, Benning C, Larkin RM. A membrane-tethered transcription factor defines 
a branch of the heat stress response in Arabidopsis thaliana. Proc Natl Acad Sci U S A. 2008; 105(42), 16398-16403.

28. Xiang Y, Tang N, Du H, Ye H, Xiong L. Characterization of OsbZIP23 as a Key Player of the Basic Leucine Zipper Transcription Factor Family for Conferring Abscisic Acid Sensitivity and Salinity and Drought Tolerance in Rice. Plant Physiol. 2008; 148(4), 1938-1952.

29. Lu G, Gao C, Zheng X, Han B. Identification of OsbZIP72 as a positive regulator of ABA response and drought tolerance in rice. Planta. 2009; 229, 605-615.

30. Orellana S, Yañez M, Espinoza A, Verdugo I, González E, Ruiz-Lara S, Casaretto JA. The transcription factor SIAREB1 confers drought, salt stress tolerance and regulates biotic and abiotic stress-related genes in tomato. Plant Cell Environ. 2010; 33(12), 2191-2208.

31. Zhu M, Meng X, Cai J, Li G, Dong T, Li Z. Basic leucine zipper transcription factor SlbZIP1 mediates salt and drought stress tolerance in tomato. BMC Plant Biol. 2018; 18(1), 83.

32. Li D, Fu F, Zhang H, Song F. Genome-wide systematic characterization of the bZIP transcriptional factor family in tomato ( Solanum lycopersicum L.). BMC Genomics. 2015; 16(1), 771.

33. Mittler R. Abiotic stress, the field environment and stress combination. Trends Plant Sci. 2006; 11(1), 15-19.

34. Wang K, Jiang S, Sun C, Lin Y, Yin R, Wang Y, Zhang M. The Spatial and Temporal Transcriptomic Landscapes of Ginseng, Panax ginseng C. A. Meyer. Sci Rep. 2015; 5, 18283.

35. Yin R, Zhao M, Wang K, Lin Y, Wang Y, Sun C, Wang Y, Zhang M. Functional differentiation and spatial-temporal co-expression networks of the NBS-encoding gene family in Jilin ginseng, Panax ginseng C.A. Meyer. PloS One. 2017; 12(7), e0181596. 
633 36. Wang Y, Li X, Lin Y, Wang Y, Wang K, Sun C, Lu T, Zhang M. Structural Variation, Functional Differentiation, and Activity Correlation of the Cytochrome P450 Gene Superfamily Revealed in Ginseng. Plant Genom. 2018; 11.

37. Kim NH, Jayakodi M, Lee SC, Choi BS, Jang W, Lee J, Kim HH, Waminal NE, Lakshmanan M, van Nguyen B, Lee YS, Park HS, Koo HJ, Park JY, Perumal S, Joh HJ, Lee H, Kim J, Kim IS, Kim K, Koduru L, Kang KB, Sung SH, Yu Y, Park DS, Choi D, Seo E, Kim S, Kim YC, Hyun DY, Park YI, Kim C, Lee TH, Kim HU, Soh MS, Lee Y, In JG, Kim HS, Kim YM, Yang DC, Wing RA, Lee DY, Paterson AH, Yang TJ. Genome and evolution of the shade-requiring medicinal herb Panax ginseng. Plant Biotechnol J. 2018 Nov; 16(11), 1904-1917. protein sequence motifs. Nucleic Acids Res. 2006; 34, W369-W373. clusters. Bioinformatics. 2013; 29(13), 1696-1697.

41. Ashburner M, Ball CA, Blake JA, Botstein D, Butler H, Cherry JM, Davis AP, Dolinski K, of gene expression data using BioLayout Express(3D). Nat Protoc. 2009; 4(10), 1535-1550. 
655

43. Nasrollahi V, Mirzaie-Asl A, Piri K, Nazeri S, Mehrabi R. The effect of drought stress on the expression of key genes involved in the biosynthesis of triterpenoid saponins in liquorice (Glycyrrhiza glabra). Phytochemistry. 2014; 103, 32-37.

44. Liu J, Wang Q, Sun M, Zhu L, Yang M, Zhao Y. Selection of reference genes for quantitative real-time PCR normalization in Panax ginseng at different stages of growth and in different organs. PloS One. 2014; 9(11), e112177.

45. Grabherr MG, Haas BJ, Yassour M, Levin JZ, Thompson DA, Amit I, Adiconis X, Fan L, Raychowdhury R, Zeng Q, Chen Z, Mauceli E, Hacohen N, Gnirke A, Rhind N, di Palma F, Birren BW, Nusbaum C, Lindblad-Toh K, Friedman N, Regev A. Full-length transcriptome assembly from RNA-Seq data without a reference genome. Nat Biotechnol. 2011; 29(7), 644-652.

46. Jin Z, Xu W, Liu A. Genomic surveys and expression analysis of bZIP gene family in castor bean (Ricinus communis L.). Planta. 2014; 239(2), 299-312.

47. Baloglu MC, Eldem V, Hajyzadeh M, Unver T. Genome-Wide Analysis of the bZIP Transcription Factors in Cucumber. PloS One. 2014; 9(4), e96014.

48. Wang J, Zhou J, Zhang B, Vanitha J, Ramachandran S, Jiang SY. Genome-wide Expansion and Expression Divergence of the Basic Leucine Zipper Transcription Factors in Higher Plants with an Emphasis on Sorghum. J Integr Plant Biol. 2011; 53(3), 212-231.

49. Syed NH, Kalyna M, Marquez Y, Barta A, Brown JW. Alternative splicing in plants - coming of age. Trends Plant Sci. 2012; 17(10), 616-623.

50. Liu J, Chen N, Chen F, Cai B, Santo SD, Tornielli GB, Pezzotti M, Cheng ZM. Genome-wide analysis and expression profile of the bZIP transcription factor gene family in grapevine (Vitis 
vinifera). BMC Genomics. 2014; 15(1), 281-281.

51. Yin Y, Zhu Q, Dai S, Lamb C, Beachy RN. RF2a, a bZIP transcriptional activator of the phloem-specific rice tungro bacilliform virus promoter, functions in vascular development. Embo J. 1997; 16(17), 5247-5259.

52. Fukazawa J, Sakai T, Ishida S, Yamaguchi I, Takahashi Y. REPRESSION OFSHOOT GROWTH, a bZIP transcriptional activator, regulatescell elongation by controlling the level of gibberellins. Plant Cell. 2000; 12(6), 901-915.

53. Chuang CF, Running MP, Williams RW, Meyerowitz EM. The PERIANTHIA gene encodes a bZIP protein involved in the determination of floral organ number in Arabidopsis thaliana. Gene Dev. 1999; 13(3), 334-344.

54. Lin Y, Wang K, Li X, Sun C, Yin R, Wang Y, Wang Y, Zhang M. Evolution, functional differentiation, and co-expression of the $R L K$ gene family revealed in Jilin ginseng, Panax ginseng C.A. Meyer. Mol Genet Genomics. 2018; 293(4), 845.

55. Todaka D, Shinozaki K, Yamaguchi-Shinozaki K. Recent advances in the dissection of drought-stress regulatory networks and strategies for development of drought-tolerant transgenic rice plants. Front Plant Sci. 2015; 6(84), 84.

56. Yoshida T, Fujita Y, Maruyama K, Mogami J, Todaka D, Shinozaki K, Yamaguchi-Shinozaki K. Four Arabidopsis AREB/ABF transcription factors function predominantly in gene expression downstream of SnRK2 kinases in abscisic acid signalling in response to osmotic stress. Plant Cell Environ. 2015; 38(1), 35-49.

57. Kang JY, Choi HI, Im MY, Kim SY. Arabidopsis basic leucine zipper proteins that mediate stress-responsive abscisic acid signaling. Plant Cell. 2002, 14(2), 343-357. 
699 58. Tang N, Zhang H, Li X, Xiao J, Xiong L. Constitutive activation of transcription factor OsbZIP46 improves drought tolerance in rice. Plant Physiol. 2012; 158(4), 1755-1768.

701 59. Ji X, Liu G, Liu Y, Zheng L, Nie X, Wang Y. The bZIP protein from Tamarix hispida, ThbZIP1, is ACGT elements binding factor that enhances abiotic stress signaling in transgenic Arabidopsis. BMC Plant Biol. 2013; 13(1), 1-13. 


\section{Figures}

A

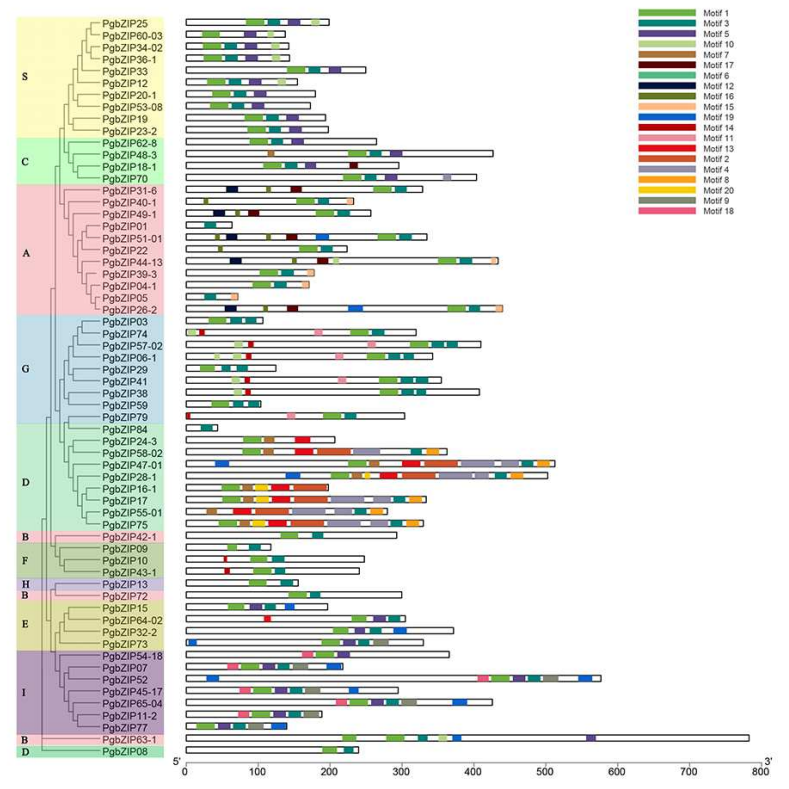

$\mathrm{B}$

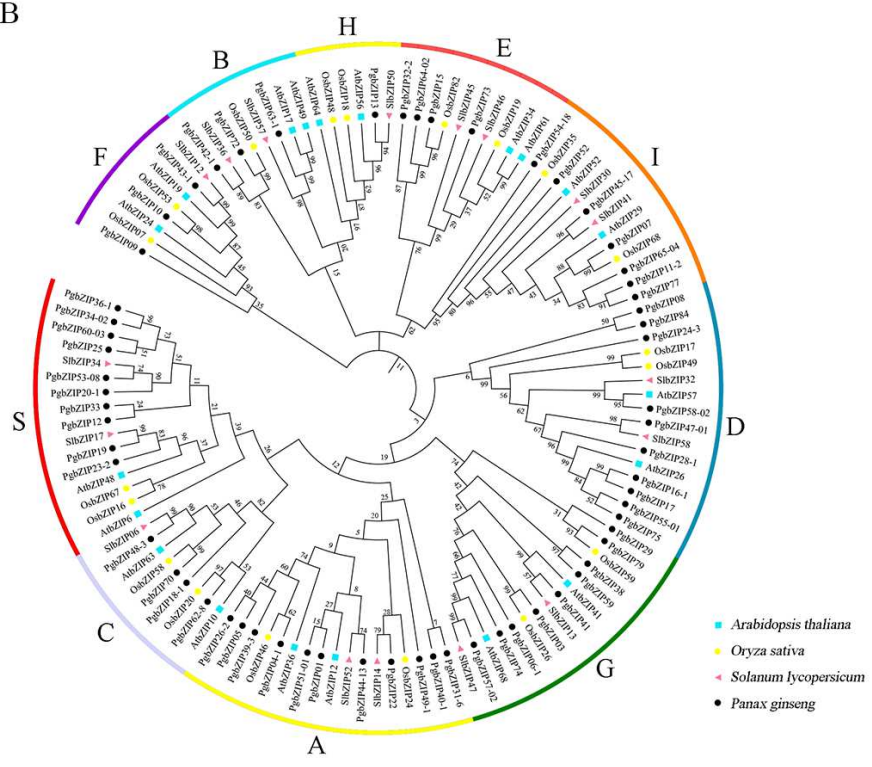

Figure 1

Conserved motifs and Phylogenetic relationship of the PgbZIP proteins. a Conserved motifs of PgbZIP proteins according to the evolutionary relationship. The conserved motifs in the PgbZIP proteins were identified with MEME software. Each motif is indicated by a colored box numbered at the top. $b$ Phylogenetic analysis of ginseng, Arabidopsis, tomato and rice bZIP proteins.

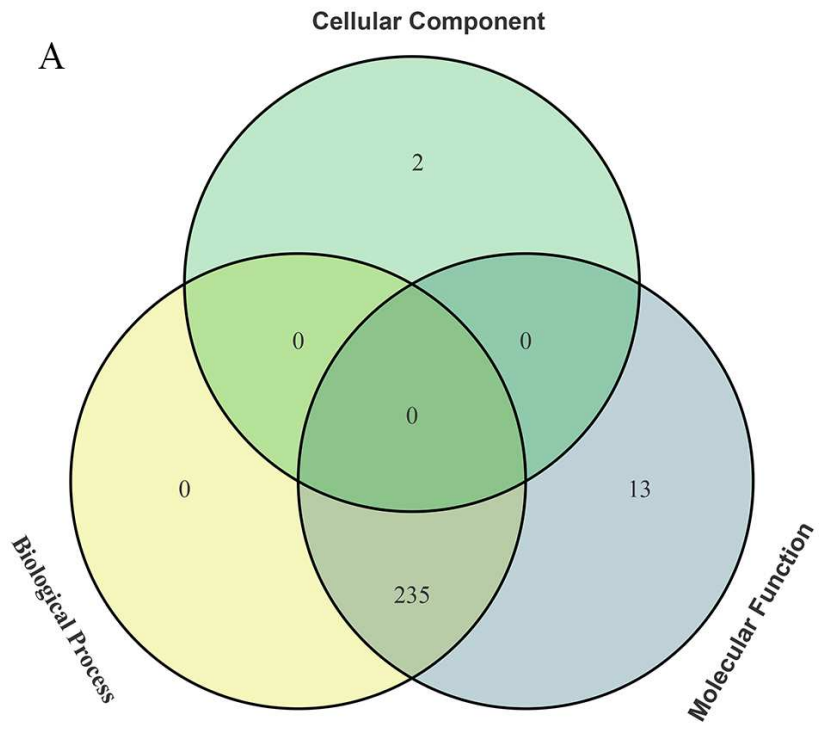

B

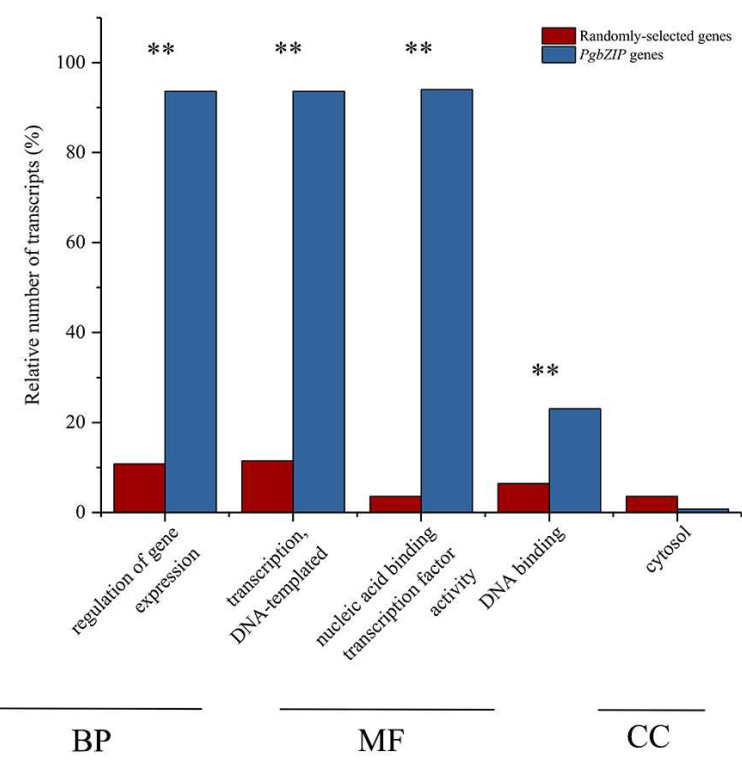


Figure 2

In silico functional categorization and GO term enrichment of the PgbZIP gene transcripts. a Venn diagram of numbers of the PgbZIP transcripts involved in the biological process (BP) (235 genes), molecular function (MF) (249 genes)and cellular component (CC) (2 genes) categories. b Subcategories in which the PgbZIP transcripts are involved at Level 2 and their enrichments. The GO terms of the transcripts expressed in 14 tissues of the four-year-old plant used for identification of the PgbZIP genes as the background control for the enrichment analysis. **, significant at $\mathrm{P} \leq 0.01$; the remaining $\mathrm{GO}$ terms are not significant at $\mathrm{P} \leq 0.05$.

A

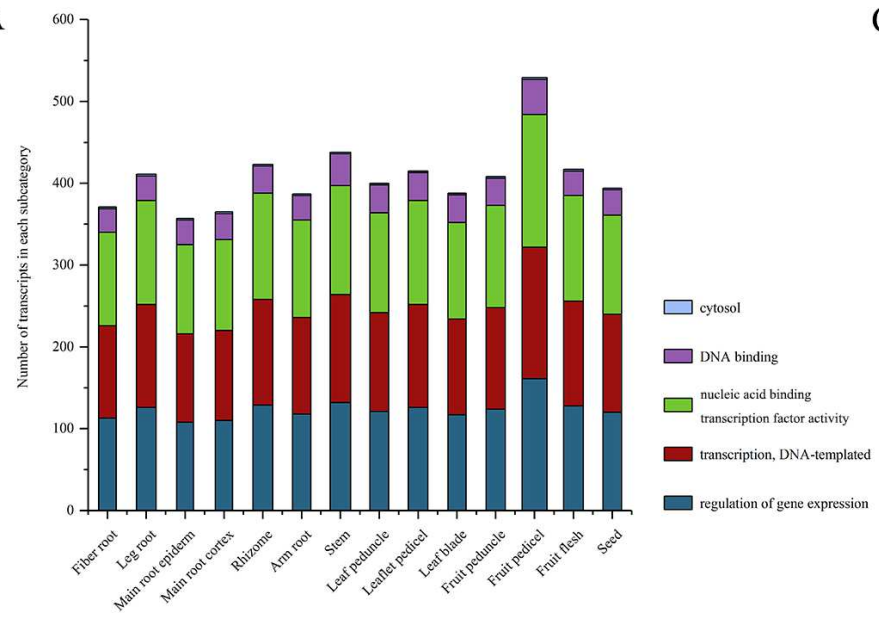

C

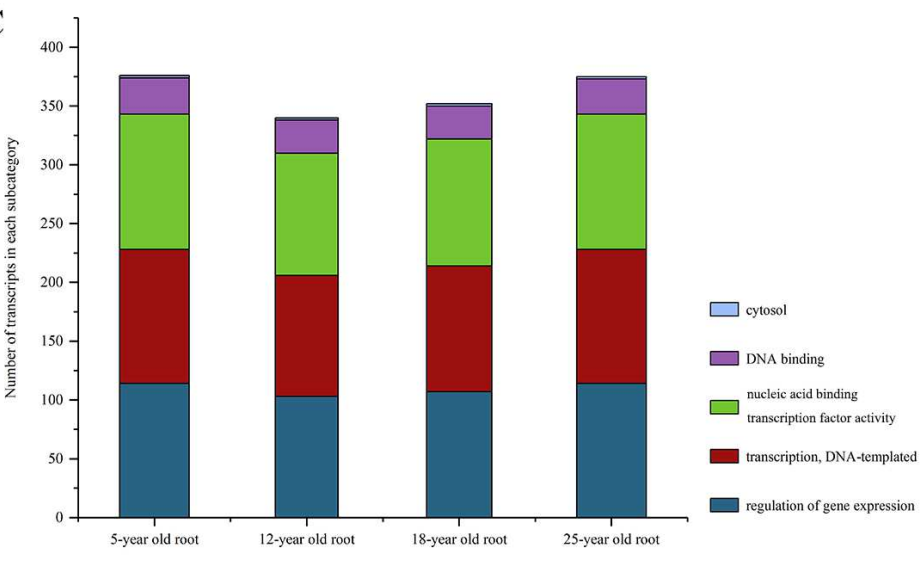

B

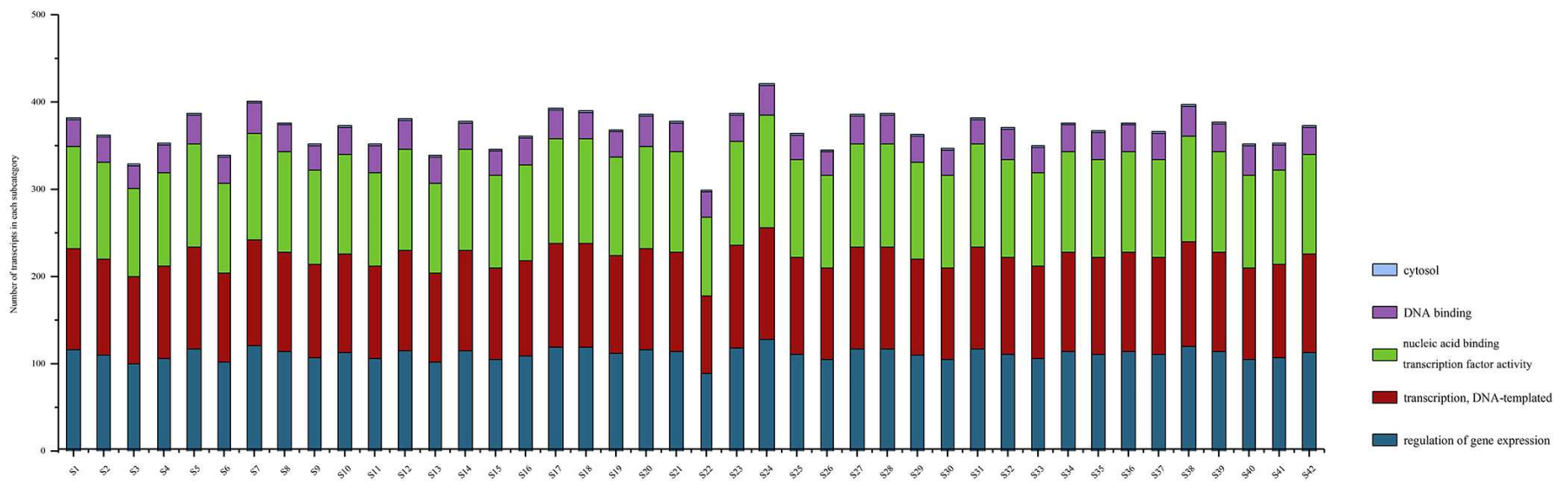

Figure 3

Variation of the functional categories of the PgbZIP transcripts. a Variation of the functional categories of the PgbZIP transcripts among 14 tissues of a 4-year-old plant. b Variation of the functional categories of the PgbZIP transcripts among the 4-year-old roots of 42 ginseng cultivars from Jilin, China. c Variation of the functional categories of the PgbZIP transcripts among the roots of differently aged plants. 


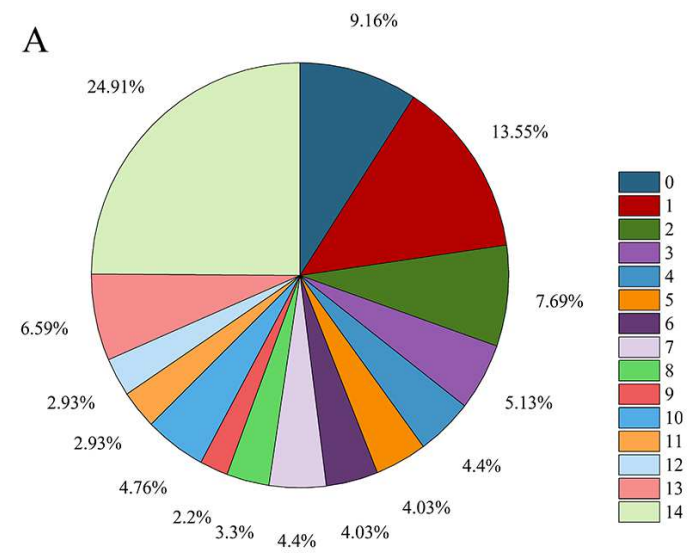

$\mathrm{C}$

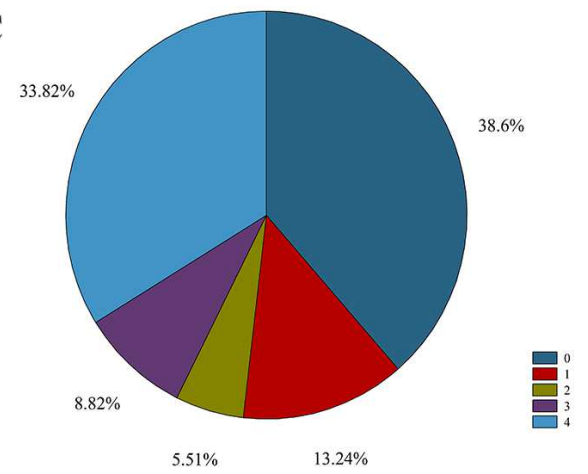

B

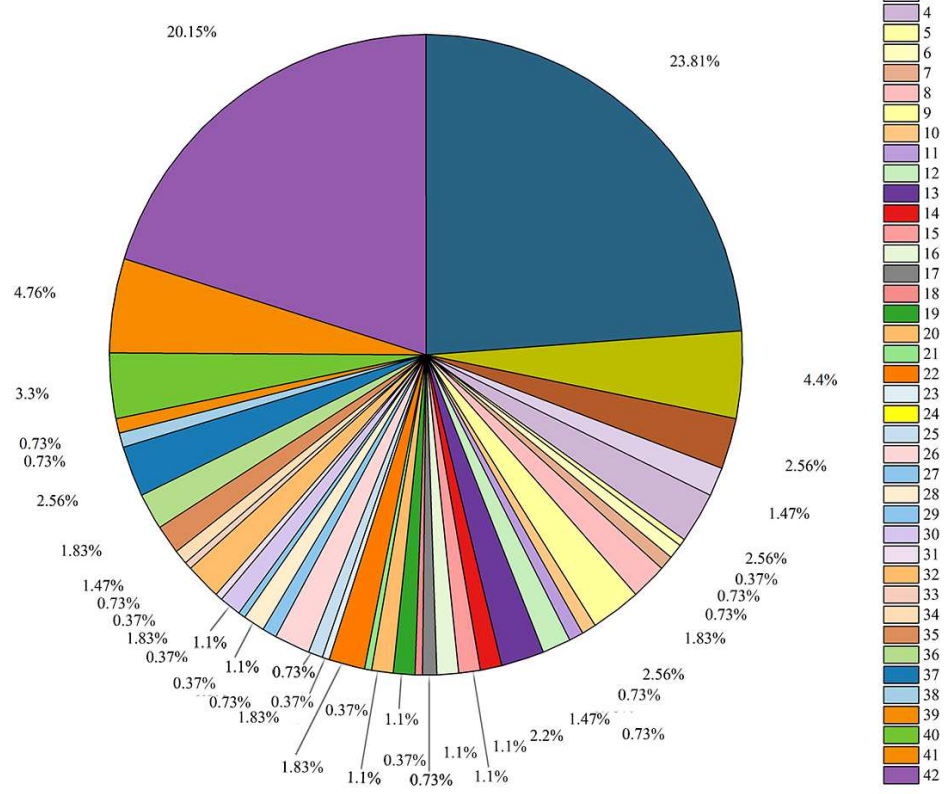

\section{Figure 4}

Expression of the PgbZIP gene transcripts in ginseng. a Expression of the PgbZIP gene transcripts in different numbers of tissues. The percentages of each part of the pie indicates the percentage of the 273 PgbZIP gene transcripts; the number behind the percentage in each part of the pie indicates the number of the 14 tissues in which the gene transcripts expressed. b Expression of the PgbZIP gene transcripts in four-year-old roots of different genotypes. The percentages of each part of the pie indicates the percentage of the 273 PgbZIP gene transcripts; the number behind the percentage in each part of the pie indicates the number of the four-year-old roots of different genotypes in which the gene transcripts expressed. c Expression of the PgbZIP gene transcripts in differently-aged roots.. The percentages of each part of the pie indicates the percentage of the 273 PgbZIP gene transcripts; the number behind the percentage in each part of the pie indicates the number of the number of four aged roots in which the gene transcripts expressed. 


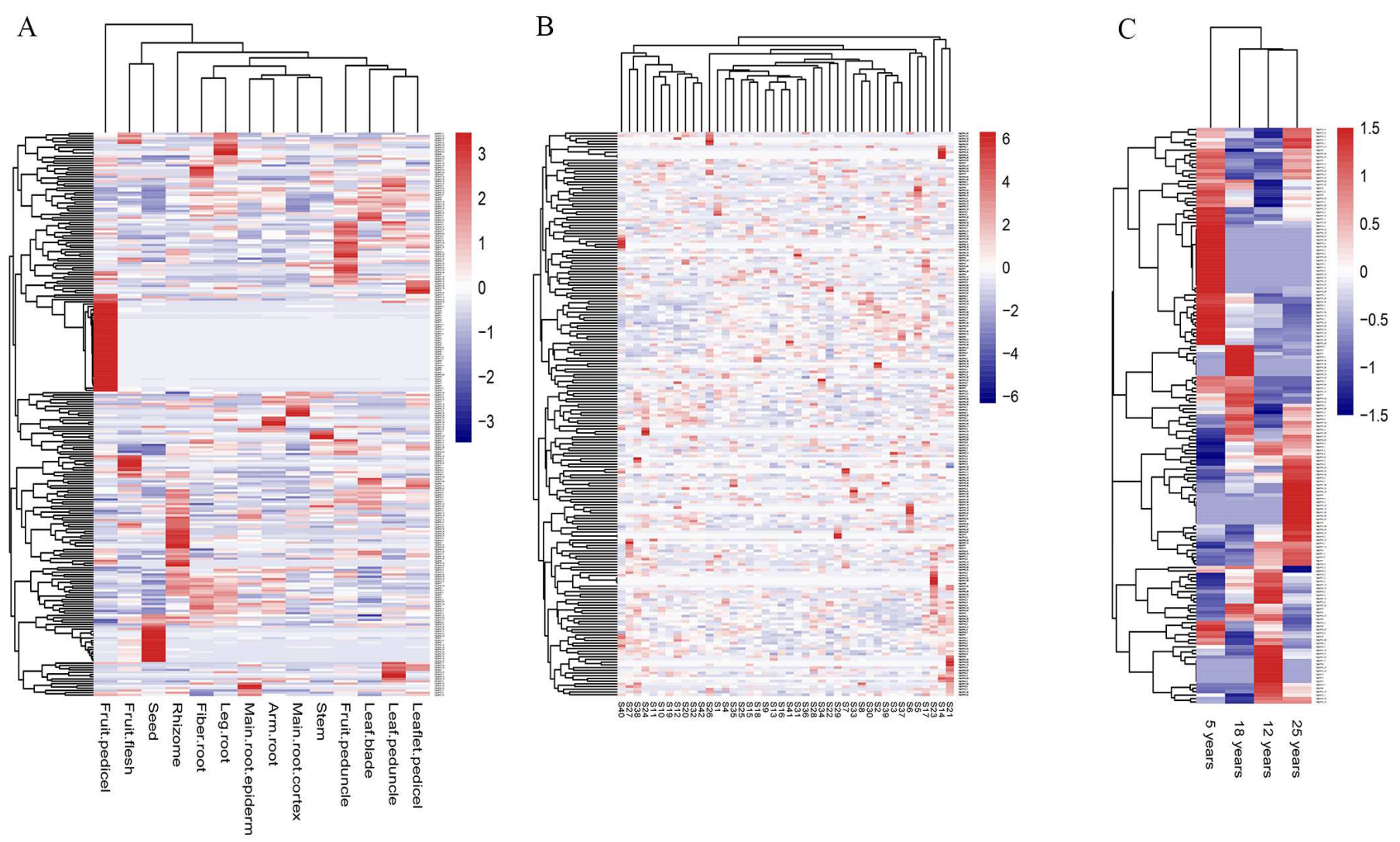

Figure 5

Expression of the PgbZIP transcripts categorized into the immune system process. a Expression of the PgbZIP transcripts in 14 tissues. b Expression of the PgbZIP transcripts in 42 cultivars. c Expression of the PgbZIP transcripts in four different year-old roots. 
A

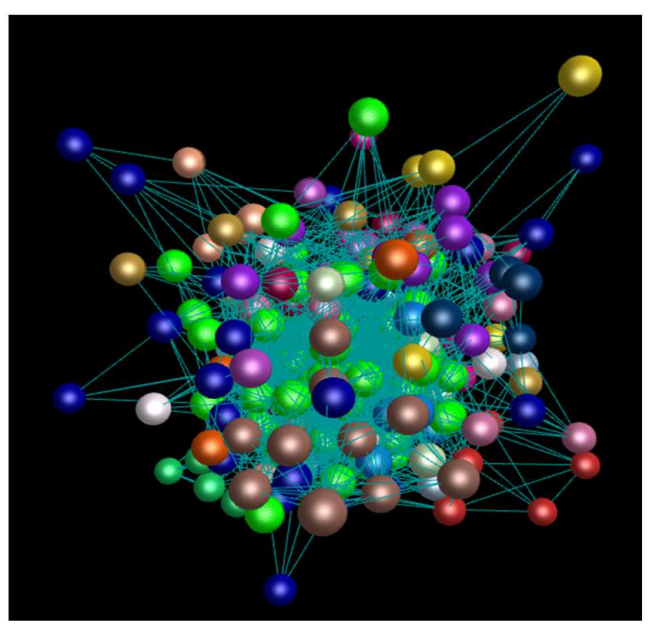

C

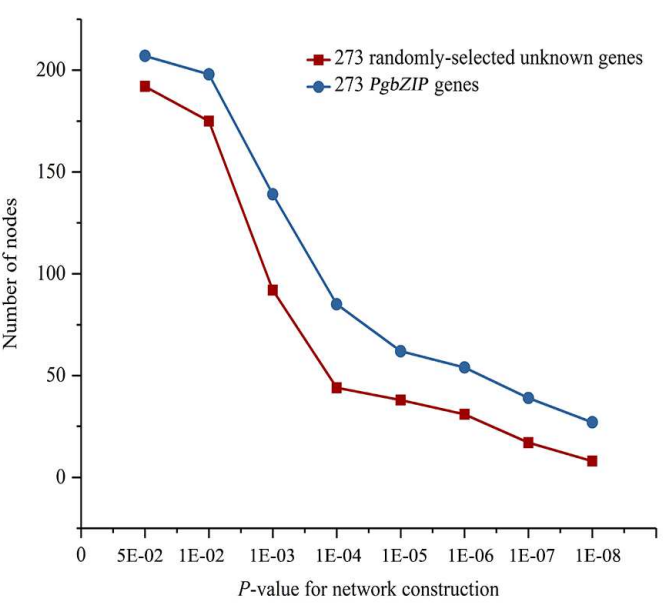

E

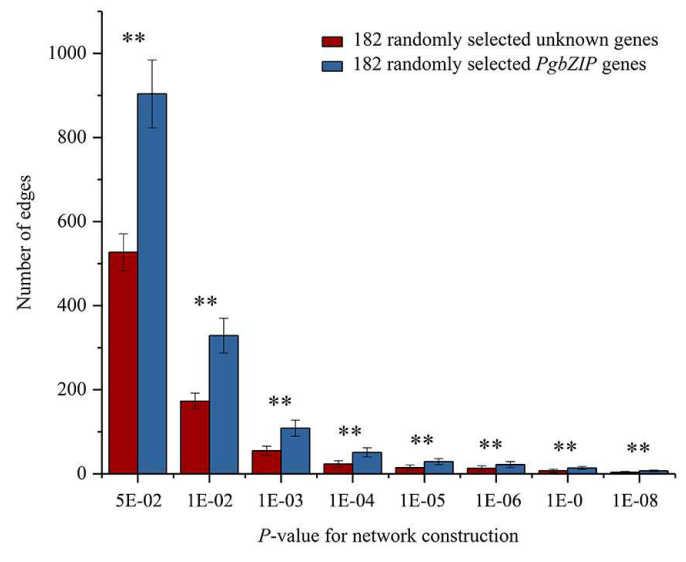

B

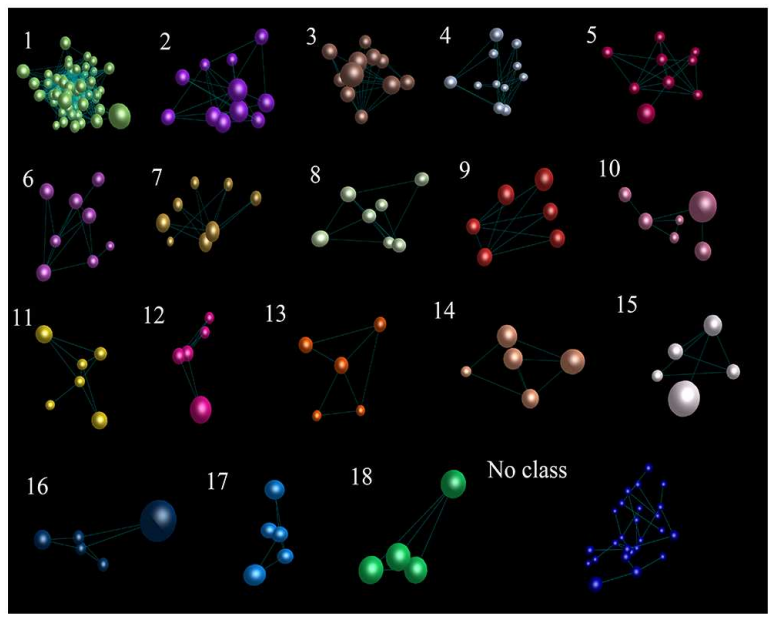

$\mathrm{D}$

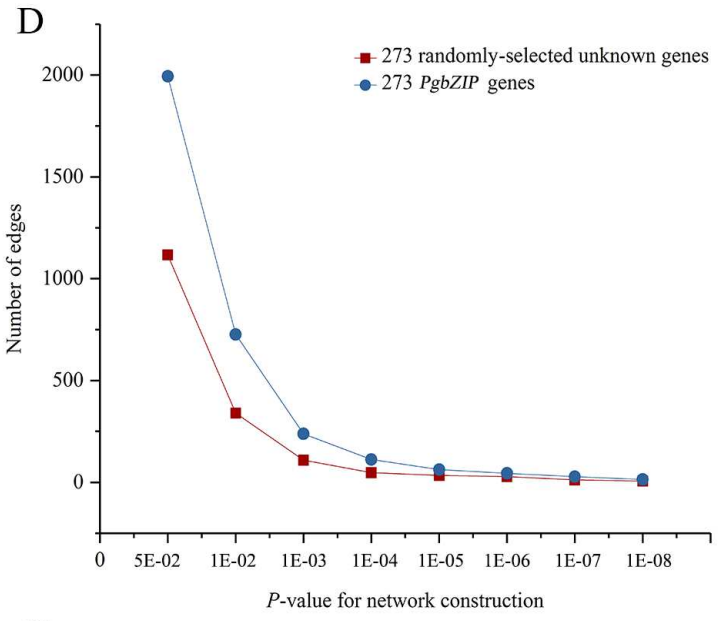

F

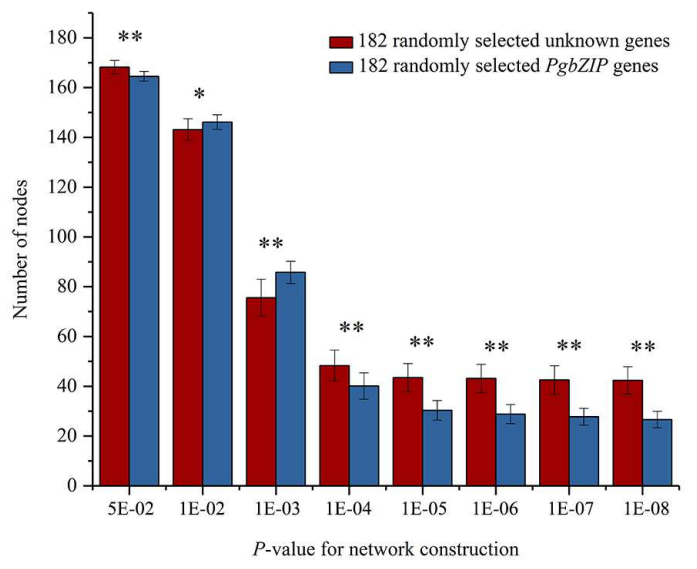

\section{Figure 6}

Network analysis of the PgbZIP transcripts expressed in the 4-year-old roots of 42 farmers' cultivars. a The co-expression network constructed from 273 PgbZIP transcripts at P $\leq 5.0 \mathrm{E}-02$. It consists of 207 nodes and 1994 edges. b 18 clusters in the network. c Tendency that PgbZIP genes form a network using the randomly-selected ginseng unknown genes as a control: variation in number of nodes. $d$ Tendency that PgbZIP genes form a network using the randomly-selected ginseng unknown genes as a control: 
variation in number of edges. The networks shown in (c and d) were constructed from 273 transcripts with no replicate. e Statistics of variation in number of nodes in the PgbZIP network. f Statistics of variation in number of edges in the PgbZIP network. Capital letters, significant at $\mathrm{P} \leq 0.01$; Error bar, the standard deviation for 20 bootstrap replications.

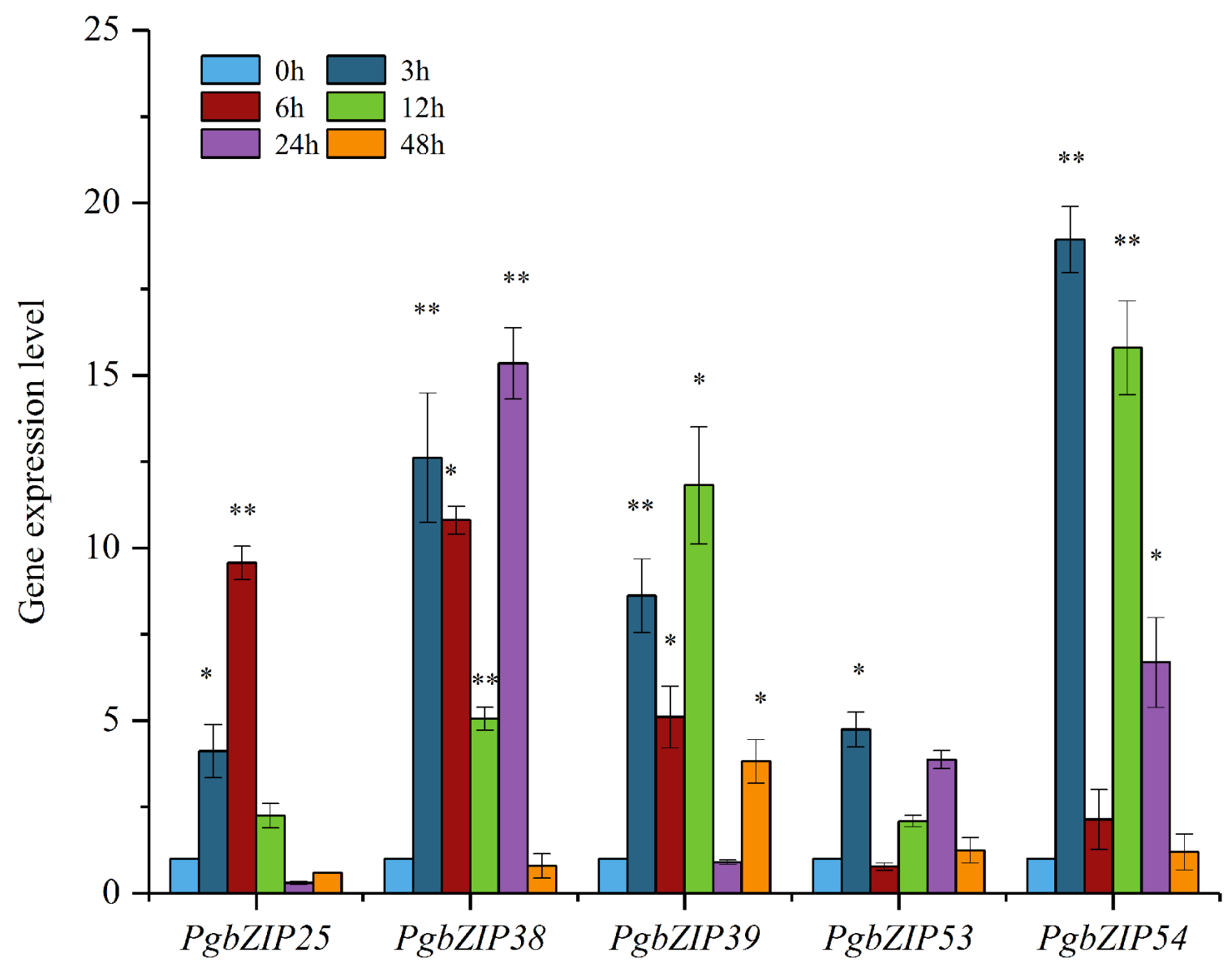

Genes

Figure 7

PgbZIP gene expression levels in ginsen seedlings after $3,6,12,24$ and $48 \mathrm{~h}$ of $20 \%$ PEG-6000 treatment. The values are expressed as the means of 3 replicates.*: $P<0.05$. **: $P<0.01$.

\section{Supplementary Files}

This is a list of supplementary files associated with this preprint. Click to download.

- S5Table.xlsx

- S1Fig.tif 
- S3Fig.tif

- S4Fig.tif

- S9Table.xlsx

- S2Fig.tif

- S8Table.xIsx

- S7Table.xIsx

- S1Table.xls

- S4Table.xlsx

- S6Table.xIsx

- S2Table.xlsx

- S3Table.xIsx 